\title{
Atherogenic index of plasma is an effective index for estimating abdominal obesity
}

\author{
Shi-Wei Shen ${ }^{1+}$, Yun Lu ${ }^{2 \dagger}$, Feng Lii ${ }^{2+}$, Cheng-Jian Yang ${ }^{1}$, Yin-Bo Feng ${ }^{2}$, Hong-Wei Li ${ }^{2}$, Wei-Feng Yao and \\ Zhen-Hai Shen ${ }^{2 *}$
}

\begin{abstract}
Background: The correlation between the atherogenic index of plasma (AIP) and waist circumference (WC) remains unknown.

Methods: A total of 5351 middle-aged men living in Southeastern China were surveyed using the random stratified cluster sampling method. A WC of $90 \mathrm{~cm}$ or greater was indicative of abdominal obesity, and AIP was calculated as follows: log [triglyceride (TG)/high-density lipoprotein-cholesterol (HDL-C)].

Results: (1) A significantly higher AIP was observed in subjects with abdominal obesity than in those without abdominal obesity $(P<0.001)$. (2) Multivariate logistic regression analysis revealed an odds ratio of 1.93, 2.59 and 2.76 for abnormal AIP levels for the second, third and fourth WC quartiles, respectively (all $P<0.001$ ) compared to the first WC quartile as a reference. (3) There was a linear correlation between WC and AIP, and a $1.0 \mathrm{~cm}$ increase in WC resulted in a 0.0175 rise in AIP. For AIP corresponding to moderate risk (0.12-0.21), WC was 85-90 cm; for AIP corresponding to high risk (> 0.21), WC was $>90 \mathrm{~cm}$.

Conclusions: AIP of $0.12-0.21$ or $>0.21$ indicates a likelihood of borderline abdominal obesity or abdominal obesity, respectively, and the combination of WC and AIP may increase the specificity and sensitivity for detection of abdominal obesity in clinical practice. The results suggest that AIP may be used as a reference to estimate abdominal obesity.
\end{abstract}

Keywords: Atherogenic index of plasma (AIP), Waist circumference, Abdominal obesity

\section{Background}

Obesity has become an important public health concern worldwide, and abdominal obesity, which involves fat accumulation in the abdomen, is recognized as an independent risk factor for obesity-related diseases and death [1]. In China, obesity has become a major risk factor for the increased prevalence of cardiovascular diseases [2]. In the present study, waist circumference (WC) was used as an index to assess abdominal obesity in middleaged men, and the correlation between WC and the atherogenic index of plasma (AIP) was evaluated to provide evidence for the development of a preventive and control strategy for atherosclerosis and cardiovascular diseases in community populations.

\footnotetext{
* Correspondence: shentaihu1954@163.com

${ }^{\dagger}$ Equal contributors

${ }^{2}$ The Taihu Rehabilitation Hospital of Jiangsu Province (Jiangsu Provincial Research Center for Health Assessment and Intervention), Wuxi, Jiangsu 214086, China

Full list of author information is available at the end of the article
}

\section{Methods}

A total of 21 survey sites (11 sites in urban areas and 10 sites in rural areas) were sampled in the three cities of Suzhou, Wuxi and Changzhou, Jiangsu Province, China, during the period from January to December 2009 using the random stratified cluster sampling method. Three villages were randomly sampled from each survey site, and approximately 200 permanent adult residents (duration of residence $>5$ years) were randomly sampled from each village. A total of 12,130 residents were investigated, of whom 11,774 had complete medical data available and were enrolled in the analysis. A total of 5351 men of Chinese Han ethnicity and aged 40-64 years underwent subsequent investigations, including 2810 (52.51\%) living in urban areas and 2541 (47.49\%) living in rural areas. Those receiving lipid-regulating drugs or with malignant tumors, Cushing's syndrome, a history of schistosome infections, severe hepatic and renal insufficiency, or incomplete medical records were excluded from this study. (Fig. 1). 


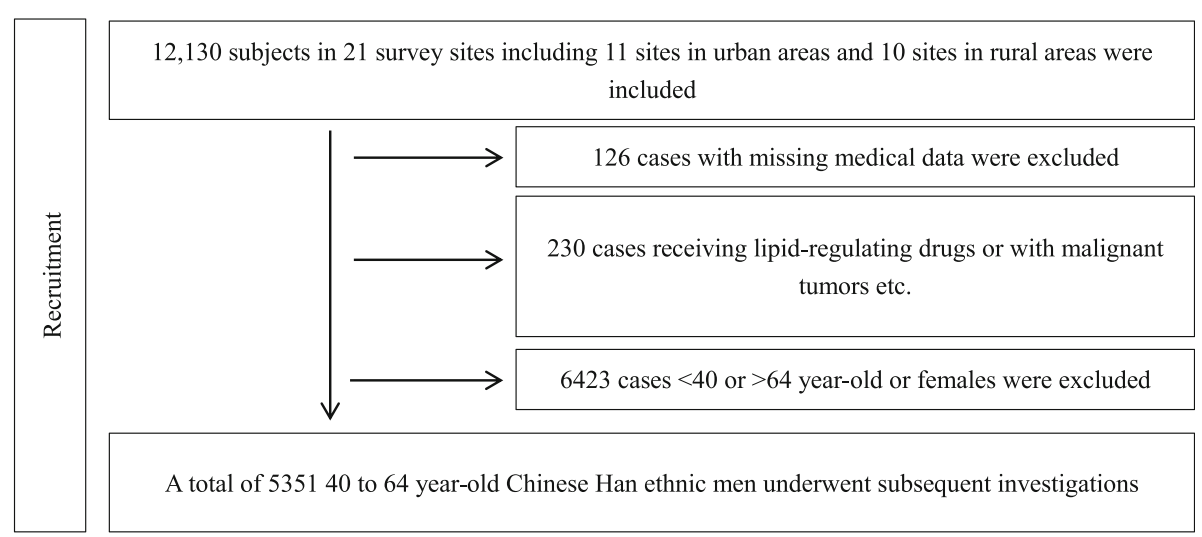

Fig. 1 Participant Flowchat

The study protocol was approved by the Ethics Review Committee of our hospital, and signed informed consent was obtained from all participants.

All data were collected using a questionnaire designed by medical professionals. Health examinations included height, body weight, WC, blood pressure measurements, and blood lipid and glucose levels. Body mass index (BMI) was calculated using the following formula: $\mathrm{BMI}=$ body weight $(\mathrm{kg}) /$ height $^{2}\left(\mathrm{~m}^{2}\right)$. WC was measured using the method recommended by the World Health Organization. In brief, each subject was instructed to stand with the feet 25-30 $\mathrm{cm}$ apart and with the back straight to allow even distribution of body weight on two legs, and then the distance between the anterior superior iliac spine and the midpoint of the inferior margin of the 12th rib was measured at the end of a normal expiration. For biochemical examinations, all participants fasted for 8-12 h before collection of $5 \mathrm{~mL}$ of venous blood from the cubital vein the following morning. Levels of total cholesterol (TC), low-density lipoprotein-cholesterol (LDL-C), high-density lipoprotein-cholesterol (HDL-C), triglyceride (TG) and fasting blood glucose (FBG) were determined using the oxidase method, a homogeneous assay, an antibody-based homogeneous assay, the glycerol phosphate oxidase method, and the hexokinase method, respectively, on a fully automatic Hitachi 7180 biochemical analyzer (Hitachi, Tokyo, Japan).

\section{Diagnostic criteria}

According to the International Diabetes Federation 2005 criteria [3], abdominal obesity was defined as WC of $\geq 90 \mathrm{~cm}$ for men. AIP was calculated as log (TG/HDL-C) [4]. AIP risk was assigned into three groups: (1) low risk, AIP $\leq 0.11$; (2) moderate risk, AIP $\geq 0.12$ and $\leq 0.21$; and (3) high risk, AIP $>0.21$ [5]. Obesity was defined as BMI of $\geq 28 \mathrm{~kg} / \mathrm{m}^{2}$ [6] and hypertension as systolic blood pressure (SBP) of $\geq 140 \mathrm{mmHg}$ and/or diastolic blood pressure
(DBP) of $\geq 90 \mathrm{mmHg}$, or currently undergoing antihypertensive therapy. Hyperglycemia was defined as FBG of $>6.1 \mathrm{mmol} / \mathrm{L}$ or currently receiving antidiabetic agents, and hyperuricemia as serum uric acid (UA) of $\geq 420 \mathrm{mmol} / \mathrm{L}$ [7]. Hypertriglyceridemia was defined as TG of $>1.7 \mathrm{mmol} / \mathrm{L}$ and hypercholesterolemia as $\mathrm{TC}$ of $>5.18 \mathrm{mmol} / \mathrm{L}$, while a HDL-C concentration of $<1.04$ $\mathrm{mmol} / \mathrm{L}$ indicated low HDL-C and a concentration of $>3.37 \mathrm{mmol} / \mathrm{L}$ indicated high LDL-C [8].

\section{Statistical analysis}

All measurement data are expressed as mean \pm standard deviation, and all statistical analyses were performed using the statistical software SPSS version 11.5 (SPSS, Chicago, IL, USA). Differences in means among groups were tested for statistical significance using the chi-square test, and associations between WC and cardiovascular risk factors were examined using multivariate logistic regression analysis and linear correlation analysis. A $P$ value $<0.05$ was considered statistically significant.

\section{Results}

\section{Comparison of cardiovascular risk factors in subjects} stratified by age and WC

Higher mean AIP, BMI, UA, SBP, DBP, TG, TC, LDL-C and FBG were observed in subjects with abdominal obesity compared to those with normal WC (all $P<0.001$ ). However, no significant difference was seen in TC level between subjects with abdominal obesity and those with normal WC aged 60-64 years $(P>0.05)$. In addition, a lower mean HDL-C concentration was noted in subjects with abdominal obesity than in those with normal WC $(P<0.001)$ (Table 1$)$.

\section{Pearson correlation analysis of WC and cardiovascular risk factors}

Pearson correlation analysis revealed that WC was positively correlated with AIP $(r=0.371)$ BMI $(r=0.786)$, UA 


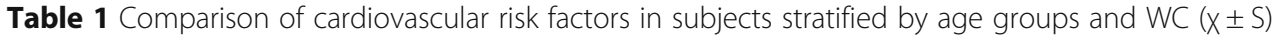

\begin{tabular}{|c|c|c|c|c|c|c|c|c|c|c|c|c|}
\hline $\begin{array}{l}\text { Age } \\
\text { (years) }\end{array}$ & $\begin{array}{l}\text { WC } \\
(\mathrm{cm})\end{array}$ & $n$ & $\begin{array}{l}\mathrm{BMl} \\
\left(\mathrm{kg} / \mathrm{m}^{2}\right)\end{array}$ & $\begin{array}{l}\text { TG } \\
(\mathrm{mmol} / \mathrm{L})\end{array}$ & $\begin{array}{l}\text { TC } \\
(\mathrm{mmol} / \mathrm{L})\end{array}$ & $\begin{array}{l}\text { HDL-c } \\
(\mathrm{mmol} / \mathrm{L})\end{array}$ & $\begin{array}{l}\text { LDL-C } \\
(\mathrm{mmol} / \mathrm{L})\end{array}$ & $\begin{array}{l}\text { UA } \\
(\mu \mathrm{mol} / \mathrm{L})\end{array}$ & $\begin{array}{l}\text { FBG } \\
(\mathrm{mmol} / \mathrm{L})\end{array}$ & $\begin{array}{l}\text { SBP } \\
(\mathrm{mmHg})\end{array}$ & $\begin{array}{l}\text { DBP } \\
(\mathrm{mmHg})\end{array}$ & AIP \\
\hline \multirow[t]{3}{*}{$40-44$} & $<90$ & 764 & $22 \pm 2.45$ & $1.92 \pm 1.63$ & $4.66 \pm 0.85$ & $24 \pm 0.36$ & $87 \pm 1.14$ & $374.76 \pm 72.72$ & $5.32 \pm 0.79$ & $124.7 \pm 13.31$ & $75.7 \pm 11.09$ & $.12 \pm 0.31$ \\
\hline & $\geq 90$ & 590 & $27.05 \pm 2.53$ & $2.69 \pm 2$ & $4.88 \pm 0.88$ & $12 \pm 0.25$ & $3.21 \pm 0.91$ & $405.85 \pm 76.77$ & $5.79 \pm 1.59$ & $133.84 \pm 15.25$ & $82.64 \pm 11.03$ & $0.31 \pm 0.31$ \\
\hline & $P$ Value & & 0.00 & 0.00 & 0.00 & 0.00 & 0.00 & 0.00 & 0.00 & 0.00 & 0.00 & 0.00 \\
\hline \multirow[t]{3}{*}{$45-49$} & $<90$ & 689 & $23.22 \pm 2.22$ & $1.88 \pm 1.58$ & $4.78 \pm 0.86$ & $1.25 \pm 0.35$ & $2.93 \pm 0.89$ & $364.98 \pm 70.34$ & $5.44 \pm 1.05$ & $125.74 \pm 13.95$ & $77.15 \pm 10.62$ & $0.11 \pm 0.31$ \\
\hline & $\geq 90$ & 661 & $26.96 \pm 2.32$ & $2.8 \pm 2.16$ & $4.94 \pm 0.9$ & $1.14 \pm 0.31$ & $3.27 \pm 0.91$ & $408.91 \pm 82.37$ & $5.91 \pm 1.41$ & $135.2 \pm 16.03$ & $83.54 \pm 10.96$ & $0.32 \pm 0.32$ \\
\hline & $P$ Value & & 0.00 & 0.00 & 0.00 & 0.00 & 0.00 & 0.00 & 0.00 & 0.00 & 0.00 & 0.00 \\
\hline \multirow[t]{3}{*}{$50-54$} & $<90$ & 587 & $23.44 \pm 2.09$ & $1.74 \pm 1.36$ & $4.62 \pm 0.88$ & $1.26 \pm 0.32$ & $2.82 \pm 0.82$ & $366.16 \pm 69.02$ & $5.61 \pm 1.39$ & 127.43 & $77.71 \pm$ & $0.08 \pm 0.3$ \\
\hline & $\geq 90$ & 538 & $26.98 \pm 2.45$ & $2.45 \pm 1.78$ & $4.82 \pm 0.94$ & $1.16 \pm 0.44$ & $3.17 \pm 0.9$ & $399.92 \pm 83.27$ & $6.09 \pm 1.62$ & $135.37 \pm 15.99$ & $83.52 \pm 10.6$ & $0.26 \pm 0.31$ \\
\hline & $P$ Value & & 0.00 & 0.00 & 0.00 & 0.00 & 0.00 & 0.00 & 0.00 & 0.00 & 0.00 & 0.00 \\
\hline \multirow[t]{3}{*}{$55-59$} & $<90$ & 467 & $23.58 \pm 2.34$ & $1.65 \pm 1.28$ & $4.64 \pm 0.86$ & $1.28 \pm 0.29$ & $2.79 \pm 0.86$ & $365.68 \pm 73.55$ & $5.65 \pm 1.26$ & $130.7 \pm 17.54$ & $78.63 \pm 11.05$ & $0.04 \pm 0.3$ \\
\hline & $\geq 90$ & 499 & $26.89 \pm 2.25$ & $2.39 \pm 2.02$ & $4.82 \pm$ & $1.16 \pm$ & 0.141 & $400.89 \pm 78.69$ & $6.12 \pm 1.5$ & $137.04 \pm 17.85$ & $82.93 \pm 11.09$ & $0.25 \pm$ \\
\hline & $P$ Value & & 0.00 & 0.00 & 0.00 & 0.00 & 0.00 & 0.00 & 0.00 & 0.00 & 0.00 & 0.00 \\
\hline \multirow[t]{3}{*}{$60-64$} & $<90$ & 275 & $23.57 \pm 2.19$ & $1.69 \pm 1.12$ & $4.72 \pm 0.77$ & $1.27 \pm 0.40$ & $2.89 \pm 0.83$ & $367.46 \pm 76.37$ & $5.7 \pm 1.03$ & $134.29 \pm 18.14$ & $79.07 \pm 11.57$ & $0.06 \pm 0.3$ \\
\hline & $\geq 90$ & 281 & $27.01 \pm 2.26$ & $2.25 \pm 1.94$ & $4.83 \pm 0.89$ & $1.17 \pm 0.24$ & $3.11 \pm 0.89$ & $391.6 \pm 89.05$ & $6.29 \pm 1.78$ & $141.99 \pm 17.84$ & $83.22 \pm 10.37$ & $0.21 \pm$ \\
\hline & $P$ Value & & 0.00 & 0.00 & 0.10 & 0.00 & 0.00 & 0.00 & 0.00 & 0.00 & 0.00 & 0.00 \\
\hline \multirow[t]{4}{*}{ Total } & $<90$ & 2782 & $23.36 \pm 2.28$ & $1.80 \pm 1.47$ & $4.68 \pm 0.86$ & $1.26 \pm 0.42$ & $2.86 \pm 0.94$ & $368.28 \pm 71.95$ & $5.50 \pm 1.11$ & $127.49 \pm 15.72$ & $77.31 \pm 11.08$ & $0.09 \pm 0.31$ \\
\hline & $\geq 90$ & 2569 & $26.98 \pm 2.38$ & $2.56 \pm 2.01$ & $4.86 \pm 0.91$ & $1.15 \pm 0.40$ & $3.19 \pm 0.91$ & $402.88 \pm 81.48$ & $6.00 \pm 1.56$ & $136.02 \pm 16.58$ & $83.17 \pm 10.86$ & $0.28 \pm 0.32$ \\
\hline & Total & 5351 & $25.10 \pm 2.95$ & $2.17 \pm 1.79$ & $4.77 \pm 0.89$ & $1.21 \pm 0.41$ & $3.02 \pm 0.94$ & $384.89 \pm 78.59$ & $5.74 \pm 1.37$ & $131.59 \pm 16.69$ & $80.12 \pm 11.36$ & $0.18 \pm 0.33$ \\
\hline & P Value & & 0.00 & 0.00 & 0.00 & 0.00 & 0.00 & 0.00 & 0.00 & 0.00 & 0.00 & 0.00 \\
\hline
\end{tabular}

$(r=0 \cdot 283)$, SBP $(r=0 \cdot 241), \mathrm{DBP}(r=0 \cdot 224), \mathrm{TG}(r=0 \cdot 266)$, TC $(r=0.139)$, LDL-C $(r=0.243)$ and FBG $(r=0.215)$ (all $P<0.001)$, and negatively correlated with HDL-C $(r=-0.222)(P<0.001)$.

\section{Multivariate logistic regression analysis of various WC quartiles and cardiovascular risk factors}

All subjects were assigned to WC quartiles: (1) first quartile (Q1), WC $\leq 84 \mathrm{~cm}$; (2) second quartile (Q2), $\mathrm{WC} \geq 85$ and $\leq 89 \mathrm{~cm}$; (3) third quartile (Q3), WC $\geq 90$ and $\leq 94 \mathrm{~cm}$; and (4) fourth quartile (Q4), WC $\geq 95 \mathrm{~cm}$. After adjustment for age, SBP, DBP, BMI, UA, TC, LDL$\mathrm{C}$ and $\mathrm{FBG}$, multivariate logistic regression analysis revealed an odds ratio of 1.93, 2.59 and 2.76 for abnormal AIP levels in Q2, Q3 and Q4 for WC (all $P<0.001$ ) with WC Q1 as the reference (Table 2).

Table 2 Multivariate logistic regression analysis of various quartiles of WC and cardiovascular risk factors

\begin{tabular}{llllll}
\hline WC group & $N$ & $\begin{array}{l}\text { Regression } \\
\text { Coefficient }\end{array}$ & $\begin{array}{l}\text { Wald chi } \\
\text { square value }\end{array}$ & OR(95\%Cl) & P值 \\
\hline Q1(WC $\leq 84 \mathrm{~cm})$ & 1463 & & & 1.00 & \\
Q2(WC85-89 cm) & 1319 & 0.66 & 27.40 & $1.93(1.51-2.46)$ & 0.00 \\
Q3(WC90-94 cm) & 1326 & 0.95 & 61.90 & $2.59(2.05-3.29)$ & 0.00 \\
Q4(WC $\geq 95 \mathrm{~cm})$ & 1243 & 1.02 & 62.83 & $2.76(2.15-3.55)$ & 0.00 \\
\hline
\end{tabular}

Notes: Age, SBP, DBP, BMI, UA, TC, LDL-C and FBG were adjusted

\section{Detection rates of abdominal obesity in various AIP quartiles}

The chi-square test revealed an increase in the detection rate of abdominal obesity with increasing AIP quartile $(P<0.001)$ : the detection rate of abdominal obesity was 2.52 times greater in AIP Q4 than in AIP Q1 (Table 3).

\section{Linear regression analysis of WC and mean AIP}

WC was classified into 27 groups (group 1, WC $\leq 75 \mathrm{~cm}$; Groups 2-26, WC $=76-100 \mathrm{~cm}$; and group 27, $\mathrm{WC} \geq 101$ $\mathrm{cm}$ ) and the mean AIP was calculated in each group. Linear regression analysis revealed a linear correlation between WC and AIP; a $1.0 \mathrm{~cm}$ increase in WC resulted in a 0.0175 rise in AIP (Fig. 2).

\section{Discussion}

Abdominal obesity, also known as central obesity, is mainly characterized by the deposition of fat in the subcutaneous abdominal region and perivisceral region. It has been shown that excessive accumulation of body fat may cause an increase in the prevalence of multiple cardiovascular risk factors, including hyperinsulinemia, insulin resistance, hypertension and dyslipidemia, which are considered the major causes of obesity-induced cardiovascular diseases [9]. We found a significantly higher AIP in subjects with abdominal obesity than in those without abdominal obesity $(t=-22 \cdot 276, P<0.001)$, and 
Table 3 Detection rates of abdominal obesity in various quartiles of AIP

\begin{tabular}{lllll}
\hline AIP groups & $\mathrm{n}(\mathrm{WC} \geq 90 \mathrm{~cm})$ & $\begin{array}{l}\text { Detection } \\
\text { Rates }(\%)\end{array}$ & $\begin{array}{l}\text { Chi square } \\
\text { value }\end{array}$ & $P$ value \\
\hline Q1 $(\leq-0.050)$ & $1337(352)$ & 26.31 & 600.831 & 0.000 \\
Q2 $(>-0.050 \leq 0.133)$ & $1338(594)$ & 44.42 & 33.210 & 0.000 \\
Q3 $(>0.133 \leq 0.389)$ & $1339(737)$ & 55.04 & 27.222 & 0.000 \\
Q4 $(>0.390)$ & $1337(886)$ & 66.26 & 283.059 & 0.000 \\
Total & $5351(2569)$ & 48.01 & 16.957 & 0.000 \\
\hline
\end{tabular}

Note: Linear-by-Linear Association 456.202, $P=0.000$

WC was positively correlated with BMI, AIP, UA, SBP, DBP, TG, TC, LDL-C and FBG, and negatively correlated with HDL-C. In addition, we found that AIP increases with WC, and a rise in AIP resulted in an increase in the detection rate of abdominal obesity. These results demonstrate that WC and AIP are parameters associated with lipid metabolism, and an increase in WC is indicative of abnormal deposition of visceral fat in the abdomen, while a rise in AIP is indicative of dyslipidemia.

Results from the Action to Control Cardiovascular Risk in Diabetes (ACCORD) study showed that among patients undergoing treatment with statins alone, the incidence of primary endpoint events was greater in those with high TG and low HDL-C levels than in other patients [10]. However, Pietro Scicchitano et al. [11] proposed functional food and nutraceuticals have the potential to become the future of primary prevention in dyslipidaemia treatment in cardiovascular disease prevention. Therefore, management of atherogenic dyslipidemia, which is characterized by hypertriglyceridemia and low HDL-C concentrations, should be a focus of attention [12].

It has been found that AIP is negatively associated with LDL particle diameter [4], and an increase in AIP indicates a rise in the proportion of small dense LDL (sdLDL) [13]. Relative to LDL, sdLDL is more likely to be oxidized and promote the production of foam cells, and sdLDL is therefore accepted as a strong risk factor for atherosclerosis and a predictive factor for emergency cardiovascular events [14]. In 2002, sdLDL was identified as a major risk factor for coronary heart disease by the National Cholesterol Education Program, and its detection was recommended by the program [15]. However, all currently available approaches used to detect sdLDL have limitations and are difficult to popularize in clinical practice. By contrast, measurement of AIP is simple, economical and feasible. Although AIP is a calculated value, it is a sensitive indicator of dyslipidemia, and may indirectly reflect the diameter of LDL-C particles [13]. We therefore hypothesized that the combination of WC and AIP may increase the specificity and sensitivity of the detection of abdominal obesity in clinical practice.

We observed a linear correlation between WC and AIP, and a $1.0 \mathrm{~cm}$ increase in WC resulted in a 0.0175 increase in AIP. When WC increased from 84 to $90 \mathrm{~cm}$, AIP increased from 0.094 to 0.203 , and AIP was $\geq 0.243$ when $\mathrm{WC}$ was $\geq 91 \mathrm{~cm}$, indicating that $\mathrm{WC}$ was $\leq 84,85-90$ and $>91 \mathrm{~cm}$ for AIP corresponding to low $(\leq 0.11)$, moderate $(0.12-0.21)$ and high risk $(>0.21)$, respectively. These results demonstrate that moderate-risk AIP indicates borderline abdominal obesity, and high-risk AIP indicates the presence of abdominal obesity.

Although WC intuitively reflects abdominal fat accumulation, it fails to quantify and differentiate visceral fat and subcutaneous fat. Considering the limitations and inconvenience of computed tomography and magnetic resonance imaging in clinical practice, a simpler way to identify abdominal obesity is of great significance for the prevention and control of cardiovascular diseases in

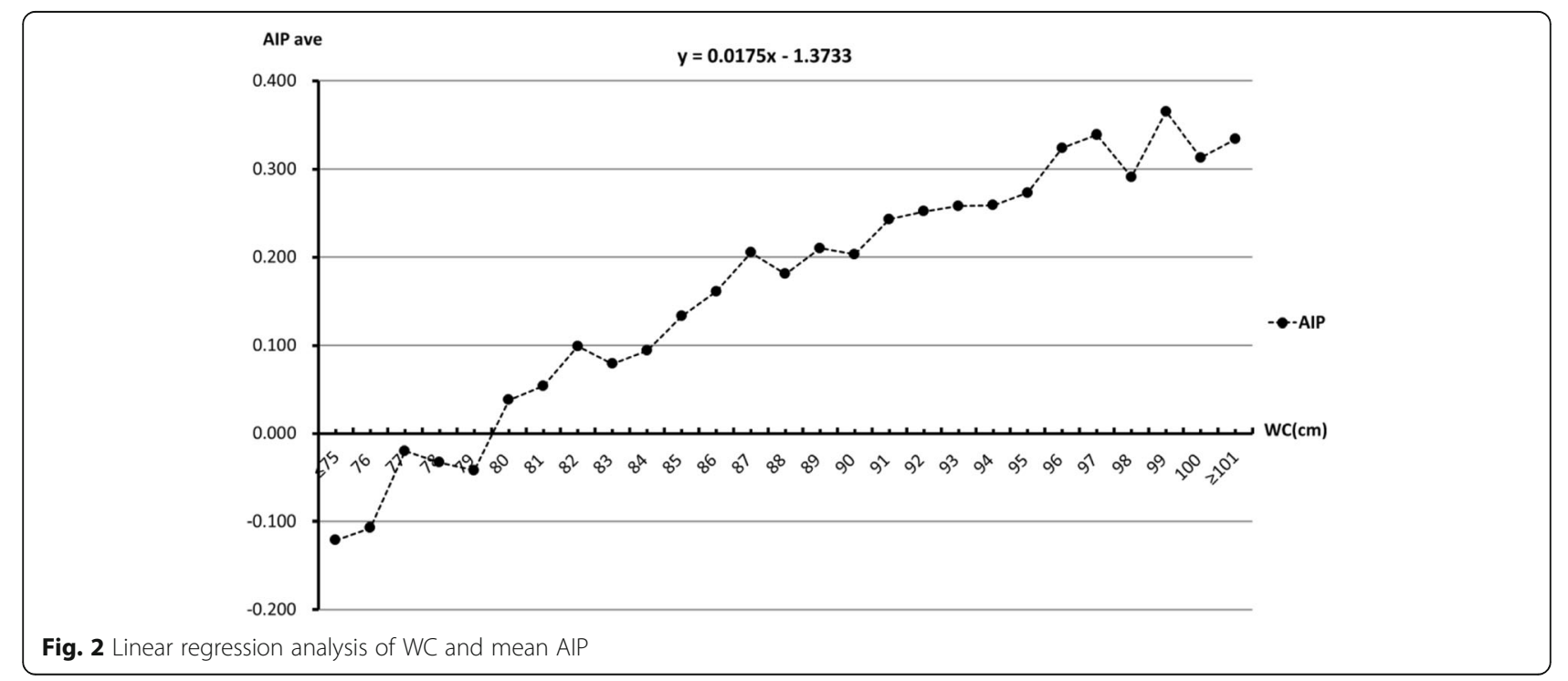


community populations. The WC cutoff point for abdominal obesity remains controversial. The World Health Organization and International Diabetes Federation recommend $\mathrm{WC} \geq 90 \mathrm{~cm}$ for men and $\geq 80 \mathrm{~cm}$ for women as the cutoff points for abdominal obesity in the Asian Pacific region [3, 16]. The Working Group on Obesity in China recommends $\mathrm{WC} \geq 85 \mathrm{~cm}$ for men and $\geq 80 \mathrm{~cm}$ for women as the cutoff points for abdominal obesity [6], while the 2011 Chinese Guidelines for Prevention of Cardiovascular Diseases recommend WC cutoff points of $\geq 90 \mathrm{~cm}$ for men and $\geq 85 \mathrm{~cm}$ for women for abdominal obesity [8]. In addition, the Japan Society for the Study of Obesity recommends WC cutoff points of $85 \mathrm{~cm}$ for men and $90 \mathrm{~cm}$ for women [17], and $85 \mathrm{~cm}$ was predicted as the WC cutoff point for insulin resistance in middle-aged Japanese men [18]. He and colleagues reported WC cutoff points of $83-85 \mathrm{~cm}$ for men and $73-76 \mathrm{~cm}$ for women in China [19], and our recent study showed that an appropriate WC cutoff point was $85 \mathrm{~cm}$ for abdominal obesity in middle-aged men living in Suzhou, Wuxi and Changzhou areas of Jiangsu Province, southeastern China [20]. In the present study we found that WC was $85-90 \mathrm{~cm}$ when AIP was 0.12-0.21, which strongly supports the previous results. Therefore, we suggest that WC cutoff points for abdominal obesity should be defined according to local epidemiological profiles.

Our study has some limitations. First, computed tomography or magnetic resonance imaging was not performed to quantitatively differentiate visceral fat and subcutaneous fat. Second, the effects of race, gender, age, region and economic levels on moderate- and high-risk AIP levels require further investigation.

\section{Conclusion}

In conclusion, moderate- or high-risk AIP indicates a likelihood of borderline abdominal obesity or abdominal obesity, respectively, and the combination of WC and AIP may increase the specificity and sensitivity of the detection of abdominal obesity in clinical practice. Our results suggest that AIP may be used as a reference to identify abdominal obesity.

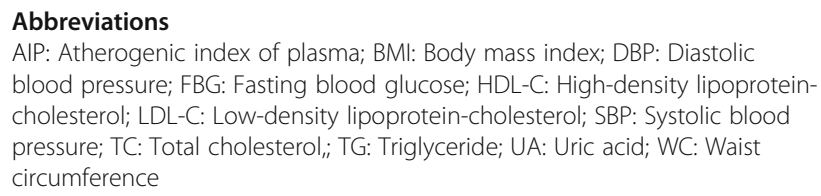

\section{Acknowledgements}

Not applicable.

\section{Funding}

The research leading to these results received funding from the National Natural Science Foundation of China (Grant No.: 81600346), Natural Science Foundation of Jiangsu Province, China (Grant No.: BK2011162 and BK20151115), the R \& D Fund of Wuxi Municipal Science \& Technology Bureau, China (Grant No.: CSE00705, CMB21S1301 and CMB41S1701), and
Jiangsu Department of Health, China (Grant Nos.: H200739, BJ14023, Z201519, BJ15032, BJ15033 and H201639).

\section{Availability of data and materials}

The datasets used and/or analyzed during the current study will be available from the corresponding author on reasonable requests after study completion.

\section{Authors' contributions}

Study conception and design: ZS, SS, YL and CY. Literature search: FL, YF, HL and WY. Data collection and analysis: FL, YF and HL. Data interpretation: ZS and SS. Writing: SS, YL and CY. All authors read and approved the final manuscript.

\section{Authors' information}

Not applicable.

\section{Ethics approval and consent to participate}

The study protocol was approved by the Ethics Review Committee of the Taihu Rehabilitation Hospital of Jiangsu Province (SG-MEC-2007-12). Written informed consent was obtained from all participants following a detailed description of the purpose of the study.

\section{Consent for publication}

Written informed consent for publication of their clinical details was obtained from the patients. Copies of the consent form are available for review by the Editor of this journal.

\section{Competing interests}

The authors report no relationships that could be construed as a conflict of interest.

\section{Publisher's Note}

Springer Nature remains neutral with regard to jurisdictional claims in published maps and institutional affiliations.

\section{Author details}

${ }^{1}$ Wuxi No.2 People's Hospital Affiliated to Nanjing Medical University, Wuxi, Jiangsu 214002, China. ${ }^{2}$ The Taihu Rehabilitation Hospital of Jiangsu Province (Jiangsu Provincial Research Center for Health Assessment and Intervention), Wuxi, Jiangsu 214086, China.

Received: 20 November 2017 Accepted: 3 January 2018 Published online: 15 January 2018

\section{References}

1. Matsuzawa Y. Establishment of a concept of visceral fat syndrome and discovery of adiponectin. Proc Jpn Acad Ser B Phys Biol Sci. 2010;86:131-41. https://doi.org/10.2183/pjab.86.131.

2. Zhu ZM. Obesity related cardiovascular risk and its appropriate intervention. Chin J Endocrinol Metab. 2011;27:707-10. https://doi.org/10.3760/cma.j.issn. 1000-6699.2011.09.002

3. Alberti KG, Zimmet P, Shaw J, IDF Epidemiology Task Force Consensus Group. The metabolic syndrome: a new worldwide definition. Lancet 2005; 366: 1059-1062. doi: https://doi.org/10.1016/S0140-6736(05)67402-8.

4. Frohlich J, Dobiasova M. Fractional esterification rate of cholesterol and triglycerides to HDL-cholesterol are powerful predictors of positive findings on coronary angiography. Clin Chem. 2003:49:1873-80.

5. Dobiasova M. Atherogenic index of plasma [log (triglycerides/HDLcholesterol)]: theoretical and practical implications. Clin Chem. 2004;50: 1113-5. https://doi.org/10.1373/clinchem.2004.033175.

6. Cooperative Meta-analysis Group of China Obesity Task Force. Predictive value of body mass index and waist circumference to risk factors of related diseases in Chinese adult population. Chin J Epidemiol. 2002;23:5-10.

7. Endocrinology Division of Chinese Medical Association. Chinese expert consensus about hyperuricemia and gout treatment. Chin J Endocrinol Metab. 2013;29:913-20. https://doi.org/10.3760/cma.j.issn.0578-1426.2017.03.021.

8. Chinese Society of Cardiology of Chinese Medical Association, Editorial Board of Chinese Journal of Cardiology. Chinese guidelines for prevention of cardiovascular diseases. Chin J Cardiol. 2011;39:3-22.

9. Carr MC, Brunzell JD. Abdominal obesity and dyslipidemia in the metabolic syndrome: importance of type 2 diabetes and familial combined 
hyperlipidemia in coronary artery disease risk. J Clin Endocrinol Metab. 2004; 89:2601-7. https://doi.org/10.1210/jc.2004-0432.

10. Scott R. Effects of combination lipid therapy in type 2 diabetes mellitus. $N$ Engl J Med. 2010;362:1563-74. https://doi.org/10.1056/NEJMoa1001282.

11. Scicchitano P, Cameli M, Maiello M, et al. Nutraceuticals and dyslipidaemia: beyond the common therapeutics. J Funct Foods. 2014;6:11-32.

12. Chinese Society of Cardiology of Chinese Medical Association. Chinese expert consensus on blood lipid related cardiovascular residual risk control. Chin J Cardiol. 2012;40:547-53

13. Dobiasova M. AIP-atherogenic index of plasma as a significant predictor of cardiovascular risk: from research to practice. Vnitr Lek. 2006;52:64-71.

14. Jia WP, Xiang KS, Chen L, Lu JX, Wu YM. Epidemiological study on obesity and its comorbidities in urban Chinese older than 20 years of age in shanghai. China Obesity reviews. 2002;3:157-65.

15. National Cholesterol Education Program (NCEP) Expert Panel on Detection, Evaluation, and Treatment of High Blood Cholesterol in Adults (Adult Treatment Panel III). Third Report of the National Cholesterol Education Program (NCEP) Expert Panel on Detection, Evaluation, and Treatment of High Blood Cholesterol in Adults (Adult Treatment Panel III) final report. Circulation. 2002; 106: 3143-3421.

16. Obesity Group of Endocrine Society of Chinese Medical Association. Expert consensus on prevention and treatment of obesity in Chinese adults. Chin J Endocrinol Metab. 2011;27:711-7. https://doi.org/10.3760/cma.j.issn.10006699.2011.09.003.

17. Examination Committee of Criteria for 'Obesity Disease' in Japan and Japan Society for the Study of Obesity. New criteria for 'obesity disease' in Japan. Circ J. 2002;66:987-92.

18. Tabata S, Yoshimitsu S, Hamachi T, Abe H, Ohnaka K, Kono S. Waist circumference and insulin resistance: a cross-sectional study of Japanese men. BMC Endocr Disord. 2009;9:1. https://doi.org/10.1186/1472-6823-9-1.

19. He Y, Zeng Q, Tian JL, Chen ZH, Zhao XL. Waist to height ratio as a predictor of dyslipidemia for Chinese adults. Chin J Health Manage. 2013;7 9-13. https://doi.org/10.3760/cma.j.jssn.1674-0815.2013.01.004.

20. Shen SW, Lu Y, Qi HJ, et al. Waist-to-height ratio is an effective indicator for comprehensive cardiovascular health. Sci Rep. 2017;7:43046. https://doi.org/ 10.1038/srep43046

\section{Submit your next manuscript to BioMed Central and we will help you at every step:}

- We accept pre-submission inquiries

- Our selector tool helps you to find the most relevant journal

- We provide round the clock customer support

- Convenient online submission

- Thorough peer review

- Inclusion in PubMed and all major indexing services

- Maximum visibility for your research

Submit your manuscript at www.biomedcentral.com/submit

) Biomed Central 